\title{
A variant of the Kleine-Levin syndrome following head trauma
}

\author{
V.S. Kostića,*, E. Stefanova ${ }^{\text {a }}$, M. Svetel ${ }^{\mathrm{a}}$ and \\ D. Kozić ${ }^{b}$ \\ a Institute of Neurology, Clinical Center of Serbia, \\ Belgrade, Yugoslavia \\ ${ }^{\mathrm{b}}$ Institute of Oncology, MRI Center, Sremska \\ Kamenica, Yugoslavia
}

\begin{abstract}
A 19-year-old man developed the Kleine-Levin syndrome three weeks after the head trauma and subsequent neurosurgical evacuation of right-sided, fronto-temporal epidural hematoma. The expression of periodic episodes was observed for hypersomnolence and, to a lesser degree, for behavioral disturbances, while the hyperphagia was constantly present during a period of 1.5 years. These clinical features were associated with the focal, right-sided hypothalamic lesion and ipsilateral posttraumatic parenchymal temporal lobe damage on NMR imaging.
\end{abstract}

Keywords: Kleine-Levin syndrome, hyperphagia, hypothalamus

\section{Introduction}

Critchley's monograph of 1962 [4] stands as the classic description of the Kleine-Levin syndrome (KLS), initially described by Kleine [11] and Levin [12]. Diagnostic criteria, modified through the experience with increasing number of complete and incomplete forms [21, 18, 16, 19], comprised episodic hypersomnolence, hyperphagia, and behavioral disorders (desinhibited behavior, mood disturbances, perplexity, cognitive impairment).

The anatomo-clinical correlations of the KLS are not fully elucidated. The presence of hypersomnia and bulimia raised the possibility of hypothalamic dysfunction $[7,8,10]$. The neuropathological studies confirmed the presence of mainly inflammatory lesions

\footnotetext{
* Corresponding author: Vladimir S. Kostić, Institute of Neurology CCS, ul. Dr Subotića 6, 11000 Belgrade, Yugoslavia. Tel.: +381 11685 554; Fax: +381 11684 577; E-mail: kostic@magelan.vet.bg.ac.yu.
}

localized in the hypothalamus, thalamic nuclei and midbrain $[22,2,6]$.

Most cases of KLS develop spontaneously, without precipitants. However, several case reports have referred to prodromal viral syndromes or episodes of encephalitis [4, 8, 14, 15, 19], as well as head trauma [23].

Herein, we report a case of 'posttraumatic' KLS with neuroradiologically proven focal unilateral hypothalamic lesion.

\section{Case report}

A 19-year-old man with no antecedent history of overt medical, neurological or psychiatric illness was admitted to the Institute of Neurology, CCS - Belgrade in April 1997 for investigation of hypersomnolence, hyperphagia and abnormal behavior.

On August 28th, 1995 he fell from $3.5 \mathrm{~m}$ when his head was injured. Following the accident he was unconscious for 'several minutes' and had retrograde amnesia for approximately the same period. Immediately after the described episode, he noticed bleeding from the right ear and since his communication ability was preserved, he presented for an examination, when this injury was managed. When he returned home he slept for an hour, woke up without headache, but vomited repeatedly for 5-6 times and fell asleep again. After 7-8 hours of 'sleep' his parents tried to wake him up, and failed to do so. In this condition, he was immediately transported to the Emergency Center of our institution where CT scan revealed epidural hematoma, frontotemporally on the right. Emergency neurosurgical evacuation of hematoma was undertaken. Forty days after the surgery the patient was comatose. After the awakening, the family failed to notice any changes of either the personality traits or his habits. However, after three weeks he started to eat indiscriminately, especially consuming huge amounts of carbohydrate 
snack, with hypersomnolence and disorganization of diurnal habits.

During the following period of 1.5 years, his parents noted periodicity of his condition in incomplete cycles, with the episodes of 6-10 weeks of deterioration and subsequent improvements of variable duration. However, these fluctuations were fairly selective: episodic nature was undoubtedly observed for hypersomnia and, to a lesser degree, for behavioral disorders, while hyperphagia was mainly persistent in the whole period, in the course of which he gained $80 \mathrm{~kg}$.

The patient was admitted to our Department in April 1997 since in the preceding weeks he had become increasingly drowsy and slept up to 16 hours daily while during the day he spent much of the time dozing on a couch. His sleep was free of apneic or snoring episodes. He was unable to maintain concentration and while watching TV, playing chess or reading, he would fall asleep within minutes. His nocturnal sleep was compromised by crying episodes, shouting or singing, which he could not recall. In a few short episodes during the day the patient was in a condition which resembled sleeping, but during the period he perceived information from the environment and was able to reproduce it subsequently. During the alertness he exhibited bizarre behavior. He would leave the Department and buy large amounts of food in the surrounding shops, while during regular hospital meals he would take leftovers of other patients. During the night he searched other patients' bedside lockers taking only food and eating it. In one of these sprees he fought with his room mate. The patient was periodically irritable and aggressive, with mood alterations between fatuousness and fearfulness. Sometimes he was suspicious with self-referential ideation. The patient never made overt sexual advances to any female patient, but he expressed increased interest in pornographic magazines. Lithium therapy ( $400 \mathrm{mg}$ b.i.d.) resulted in improvement of his hypersomnolence and irritability, while hyperphagia persisted.

Somatically, the patient was $184 \mathrm{~cm}$ tall, weighting $146 \mathrm{~kg}$. Neurologically, prosody was disturbed. His vision was possibly traumatically impaired on the right eye (visual acuity $\mathrm{OD}=0.7$; $\mathrm{OS}=1.0$ ) and the left homonymous hemianopsia was evidenced (Fig. 1). All hematological, biochemical, hormonal and cerebrospinal fluid investigations were normal. Serial electroencephalography (EEG), including EEG with nasopharyngeal leads and sleep was normal. During the neuropsychological testing the patient was cooperative, although he made several inappropriate with-

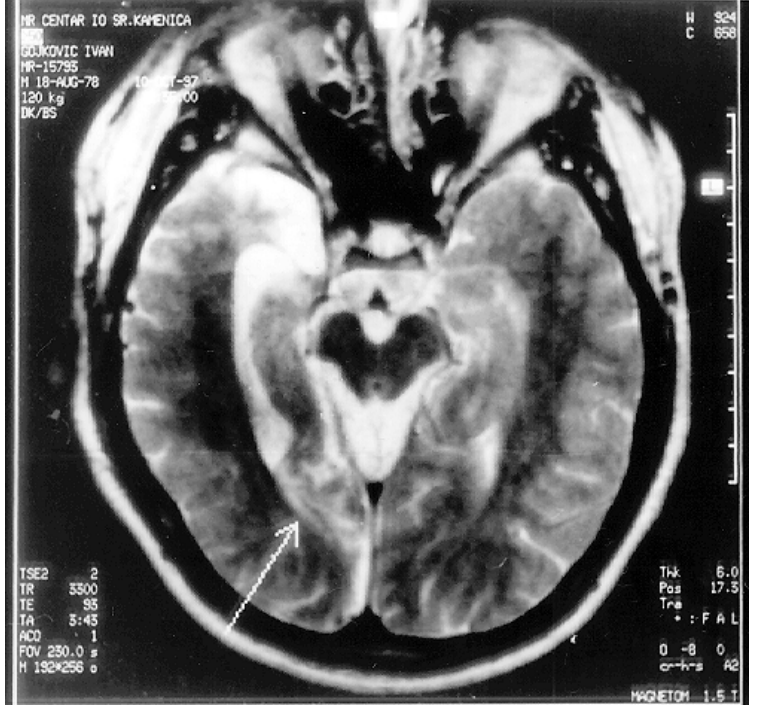

Fig. 1. A T2 weighted MR image shows high intensity lesion in the right occipital lobe (arrow).

drawals from testing in order to eat or sleep. $\mathrm{He}$ fell asleep several times during the testing procedure. The melodic quality of his speech production was disturbed, with significant change in intonation patterns of his expressive language. He produced funny and witty remarks, never going beyond the socially acceptable behavioral limits. He was of average premorbid intelligence (data obtained from his school psychologist), with current 19 points discrepancy in his Verbal $(\mathrm{VIQ}=108)$ and Performance Intelligence Quotients $(\mathrm{PIQ}=89)$ from the Wechsler Adult Intelligence Scale - Revised form [WAIS-R], revealing significant difference in comparison to premorbid state only for the PIQ. Unfortunately, we were unable to obtain a precise measure of his premorbid IQ, but, according to his marks at school he was in the upper $30 \%$. The borderline or mild declarative episodic memory deficits on immediate and delayed recall (Wechsler Memory Scale - Revised form [WMS-R], Rey Auditory Verbal Learning Test [RAVLT], Rey-Osterieth Complex Figure Test [R-OCFT]) were in contrast to intact recognition. Slight predominance in memory deficits was obtained for visual modality. Procedural memory (Tower of Toronto, Serial Reaction Time Test), executive functioning (Wisconsin Card Sorting Test), visuospatial and visuoconstructive abilities (R-OCFT, Hooper Visual Organization Test) were intact. The moderate decline on visuomotor tracking and scanning performance (Trail Making Test form A and B, digit symbol, picture completion of WAIS-R, visual span on WMS$\mathrm{R}$ ) was observed. The described alteration of prosody 


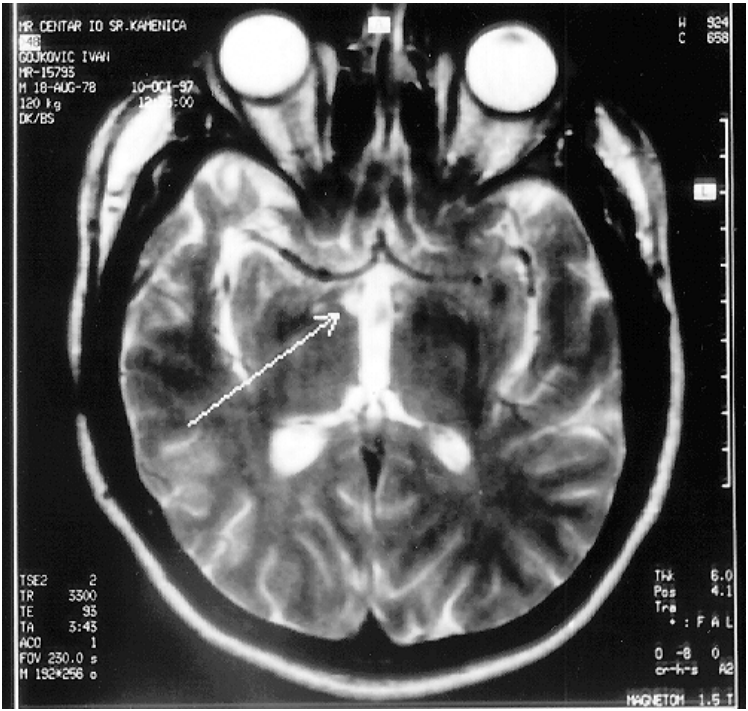

Fig. 2. A T2 weighted MR image shows high intensity lesion in the right hypothalamus (arrow) and in the right temporal lobe.

could be categorized as motor aprosody. No evidence of aphasia, apraxia and agnosia was found.

NMR imaging showed a lesion in the right hypothalamus (Fig. 2) with posttraumatic parenchymal defect of the right temporal lobe with a minor damage of the right occipital lobe.

\section{Discussion}

The patient described in this report partially fulfills the diagnostic criteria for the KLS [4]. Head trauma has been reported as antecedent of KLS [4, 23]. The close temporal relationship of the head injury to the onset of symptoms in our patient (3 weeks), as in two of the patients in Critchley's review (1962) and in two cases reported by Will et al. [23], raises a possibility that trauma precipitated KLS. However, in both patients described by Will et al. [23] CT head scans did not reveal any morphological damage. The NMR imaging study of our patient (Fig. 2) showed unilateral posttraumatic hypothalamic lesion, as well as the ipsilateral parenchymatous damage of the temporal lobe. The hypothalamic region has been already focused as a part of the neuropathological substrate of $\operatorname{KLS}[6,22]$ : pathological examination provided evidence of perivascular inflammatory infiltrates and microglial proliferation in the hypothalamus, and in particular, on the floor of the third ventricle in one case of KLS with localized encephalitis [6], as well as neuronal loss, glial proliferation and perivascular lympho- cytic cuffing of this region in a case reported by Takrani and Cronin [22]. Contrary to these data, pathological examination in Carpenter's case (1982) disclosed normal hypothalamic region, as in the CT studies of Will et al. [23]. Herein we report a case whose clinical symptoms of KLS were associated with defined, neuroradiologically proven, unilateral hypothalamic lesion (Fig. 2).

The KLS is an episodic disorder, while the expression of periodicity of the symptoms in our patient was only partial: episodic appearance was observed for hypersomnolence, and, to a lesser degree, for behavioral disturbances, but hyperphagia was constantly present during a period of 1.5 years. Pike and Stores [17] described the case of a boy with KLS and the initial absence of a clear cyclical pattern, which obscured the diagnosis. However, in our case contribution of the right-sided lesion of the temporal lobe to the clinical manifestations cannot be ruled out. The selective decline in the PIQ, the mild episodic visual memory deficits, difficulties on visuomotor tracking tasks, and aprosody could be considered as posttraumatic neuropsychological sequels, and could be explained with predominant posttraumatic right hemispheric lesion [5, 9]. Organic hyperphagia is an uncommon condition that occurs in KLS, Kluver-Bucy syndrome, Prader-Willi syndrome, hypothalamic and, occasionally, in thalamic lesions [1]. The human Kluver-Bucy syndrome has been divided into six component symptoms: visual agnosia, placidity, altered sexual behavior, hyperorality, altered dietary habits (the only symptom from this group undoubtedly present in our patient) and hypermetamorphosis, and a minimum of three of these symptoms was required for the diagnosis of the partial form of this syndrome [13]. Even in the partial syndrome, the characteristic behavior cluster of the Kluver-Bucy syndrome was thought to be diagnostic of bilateral temporal damage. However, Bates and Sturman [1] reported a woman with hyperphagia, placidity and hyposexuality associated with gross atrophy of her right temporal lobe following neurosurgery.

Finally, it is possible that trauma precipitated the KLS in our patient, with characteristic periodic episodes of hypersomnolence and behavioral symptoms, while the continuity of hyperphagia may be the consequence of the focal hypothalamic lesion (Fig. 2). Hyperphagia and the problems of driven food-seeking behavior have been described following posttraumatic hypothalamic lesion [3] or with hypothalamic damage following surgery for craniopharyngioma [20]. 


\section{References}

[1] G.D.L. Bates and S.G. Sturman, Unilateral temporal lobe damage and the partial Kluver-Bucy syndrome, Behavioral Neurology 8 (1995), 103-107.

[2] S. Carpenter, R. Yassa and R. Ochs, A pathologic basis for Kleine-Levin syndrome, Archives of Neurology 39 (1982), 25-28.

[3] A. Childs, Naltrexone in organic bulimia: a preliminary report, Brain Injury 1 (1987), 49-55.

[4] M. Critchley, Periodic hypersomnia and megaphagia in adolescent males, Brain 85 (1962), 627-656.

[5] S. Dikmen, R. Reitan and N.R. Temkin, Neuropsychological recovery in head injury, Archives of Neurology 40 (1983), 333-338.

[6] F. Fenzi, A. Simonati, F. Crosato, L. Ghersini and N. Rizzuto, Clinical features of Kleine-Levin syndrome with localized encephalitis, Neuropediatrics 23 (1993), 292-295.

[7] A. Gallinek, Syndrome of episodes of hypersomnia, bulimia, and abnormal mental states, Journal of American Medical Association 154 (1954), 1081-1083.

[8] H. Garland, D. Sumner and P. Fourman, The Kleine-Levin syndrome: some further observations, Neurology (Cleveland) 15 (1965), 1161-1167.

[9] G.M. Geffen, P. Butterworth, G.M. Forrester and L.B. Geffen, Auditory verbal learning test components as measures of severity of closed head injury, Brain Injury 5 (1994), 405411.

[10] N.N. Iakhno, Kleine-Levin syndrome, Soviet Medicine 5 (1980), 109-111.

[11] W. Kleine, Periodische Schlafsucht, Monatsschrift für Psychiatrie und Neurologie 57 (1925), 285-298.

[12] M. Levin, Periodic hypersomnolence and morbid hunger - a new syndrome, Brain 59 (1936), 494-504.
[13] R. Lilly, J.L. Cumming, F. Benson and M. Frankel, The human Kluver-Bucy syndrome, Neurology (Cleveland) $\mathbf{3 3}$ (1983), 1141-1145.

[14] R. Markman, Kleine-Levin syndrome: report of a case, American Journal of Psychiatry 123 (1967), 1025-1026.

[15] A.E. Merriam, Kleine-Levin syndrome following acute viral encephalitis, Biological Psychiatry 21 (1986), 1301-1304.

[16] M.J. Orlosky, The Kleine-Levin syndrome: a review, Psychosomatics 23 (1982), 609-621.

[17] M. Pike and G. Stores, Kleine-Levin syndrome: a cause of diagnostic confusion, Archives of Disease in Childhood $\mathbf{7 1}$ (1994), 355-357.

[18] B. Roth and S. Nevsimalova, The clinical picture of periodic hypersomnia: a study of 38 personally observed cases, in: Sleep, W.P. Koella, ed., Karger, Basel, 1980, pp. 120-124.

[19] M.S. Salter and P.D. White, A variant of the Kleine-Levin syndrome precipitated by both Epstein-Barr and VaricellaZoster virus infections, Biological Psychiatry 33 (1993), 388390.

[20] A. Skorzewska, S. Lal, J. Waserman and H. Guyda, Abnormal food-seeking behavior following surgery for craniopharyngioma, Neuropsychobiology 21 (1989), 17-20.

[21] Y. Takahashi, Clinical studies of periodic somnolence. Analysis of 28 personal cases, Psychiatria et Neurologia Japonica 67 (1965), 853-889.

[22] L.B. Takrani and O. Cronin, Kleine-Levin syndrome in a female patient, Canadian Psychiatric Association 21 (1967), 315-318.

[23] R.G. Will, J.P.R. Young and D.J. Thomas, Kleine-Levin syndrome: report of two cases precipitated by head trauma, British Journal of Psychiatry 152 (1988), 410-412. 


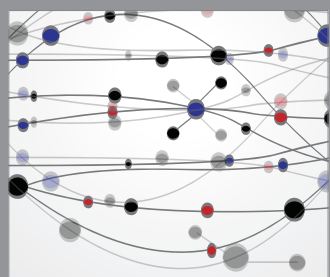

The Scientific World Journal
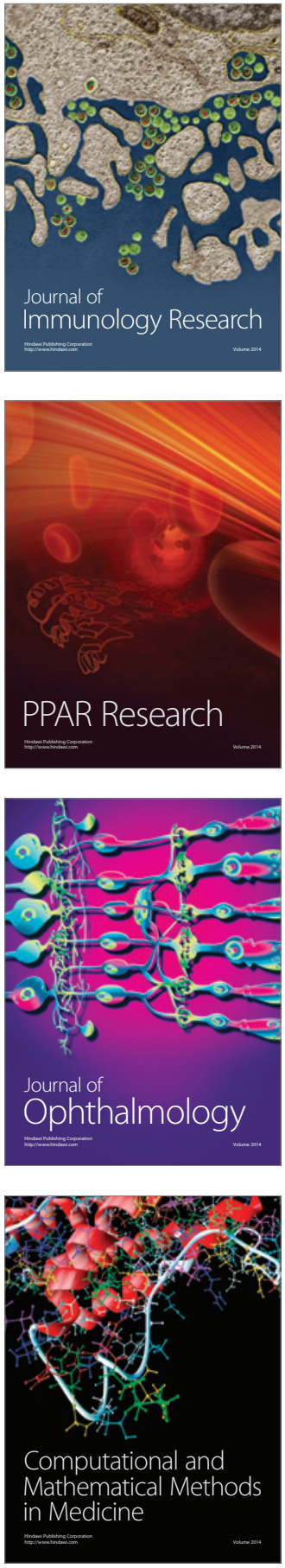

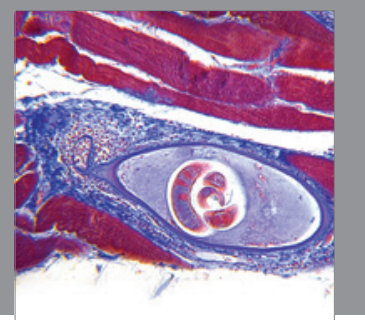

Gastroenterology

Research and Practice
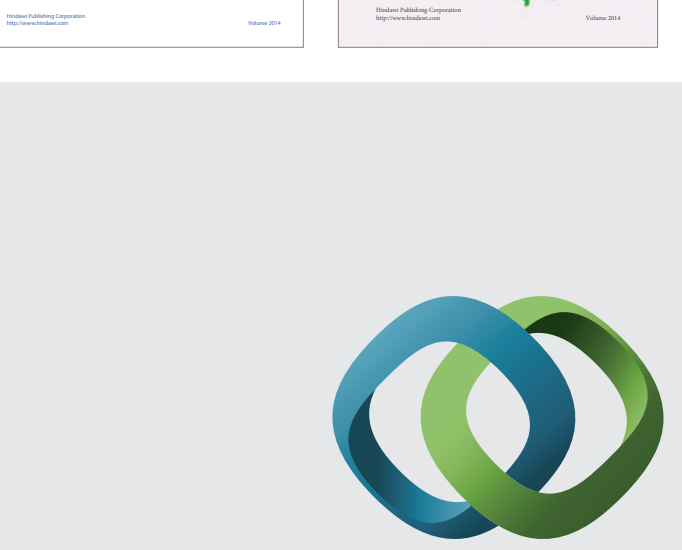

\section{Hindawi}

Submit your manuscripts at

http://www.hindawi.com
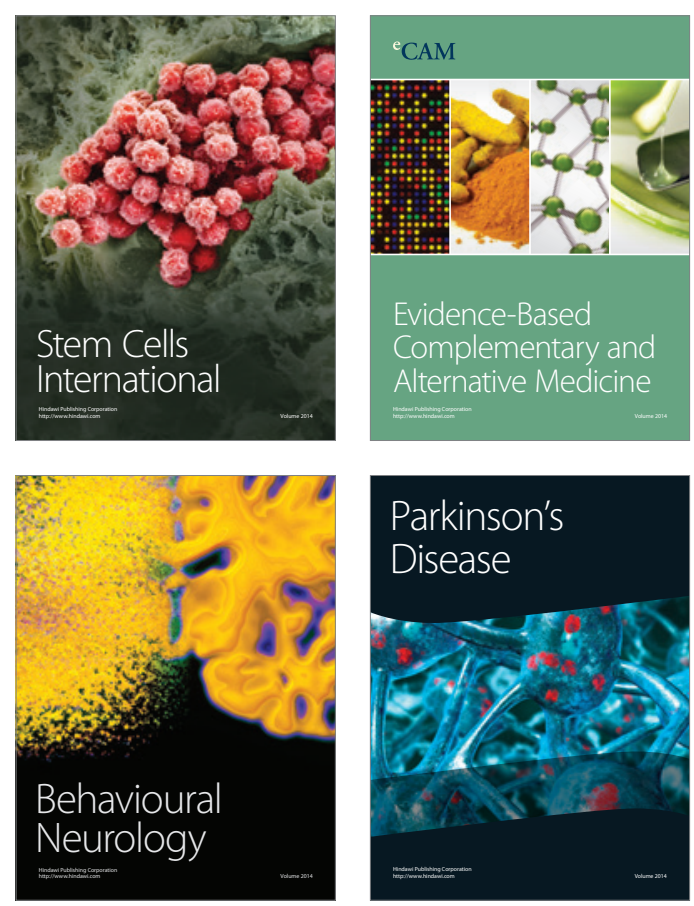

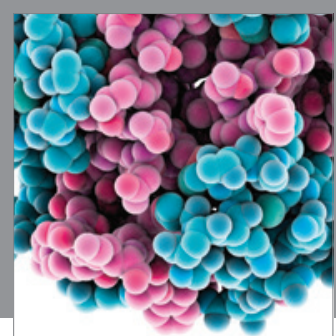

Journal of
Diabetes Research

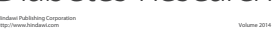

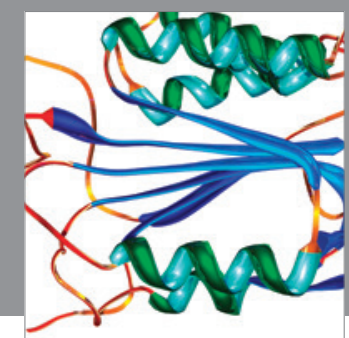

Disease Markers
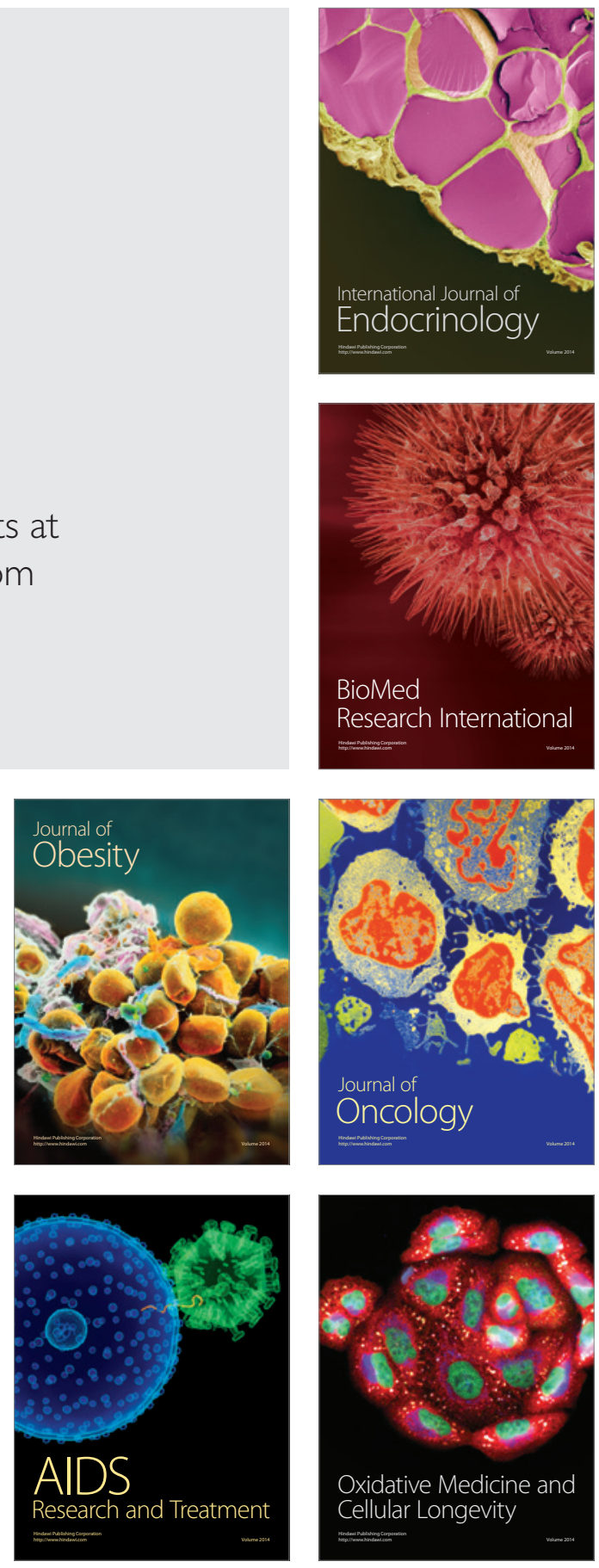